\title{
Predicting solvent effects on the 1-dodecene hydroformylation reaction equilibrium
}

\author{
Max Lemberg ${ }^{1}$, Martin Gerlach ${ }^{2}$,Emilija Kohls ${ }^{3}$, Christof Hamel ${ }^{2,4}$, Andreas \\ Seidel-Morgenstern ${ }^{2,5}$, Matthias Stein ${ }^{3}$, Gabriele Sadowski ${ }^{1, *}$
}

${ }^{1}$ TU Dortmund University, Department of Biochemical and Chemical Engineering, Laboratory of Thermodynamics, Emil-Figge-Straße 70, D-44227 Dortmund

${ }^{2}$ Otto-von-Guericke-University Magdeburg, Institute of Process Engineering, Universitätsplatz 2, D-39106 Magdeburg

${ }^{3}$ Max-Planck-Institute for Dynamics of Complex Technical Systems, Molecular Simulations and Design Group, Sandtorstraße 1, D-39106 Magdeburg

${ }^{4}$ Anhalt University of Applied Sciences, Department of Bioscience and Process Engineering, Bernburger Straße 55, D-06366 Köthen

${ }^{5}$ Max-Planck-Institute for Dynamics of Complex Technical Systems, Physical and Chemical Foundations of Process Engineering, Sandtorstraße 1, D-39106 Magdeburg

*Gabriele.Sadowski@bci.tu-dortmund.de 
TOPICAL HEADING: Thermodynamics and Molecular-Scale Phenomena

KEYWORDS: Gas solubility, Reaction equilibrium, Hydroformylation, Enthalpy of reaction, Gibbs energy of reaction

\section{ABSTRACT}

This work investigates solvent effects on the reaction equilibrium of the 1-dodecene hydroformylation in a decane/N,N-dimethylformamide solvent system. The reaction was performed at different decane $/ N, N$-dimethylformamide ratios and at temperatures between $368 \mathrm{~K}$ and $388 \mathrm{~K}$. The equilibrium concentrations of all reactants and products were determined experimentally. The enthalpy and Gibbs energy of this reaction at the ideal-gas standard state were determined by quantum-chemical calculations in good agreement with literature data. Moreover, quantum-chemically calculated standard Gibbs energies of reaction at infinite dilution in liquid decane/DMF-solvent mixtures allowed a qualitative prediction of the solvent effect on the equilibrium concentrations.

Based on the standard Gibbs energy of reaction at the ideal-gas standard state and on fugacity coefficients calculated using the Perturbed-Chain Statistical Associating Fluid Theory (PCSAFT), the equilibrium concentrations of reactants and products for the 1-dodecene hydroformylation performed in decane $/ N, N$-dimethylformamide mixtures of different compositions could be predicted in very good agreement with experimental data.

\section{INTRODUCTION}

Solvent effects on reactions were discovered and studied experimentally already in the 19th century. A comprehensive overview of studies on solvent effects is e.g. given by Reichardt ${ }^{1}$ 
which covers both, solvent effects on reaction equilibria and on reaction kinetics. Moreover, Reichardt presented a theoretical background based on the Gibbs energy of solvation that allowed correlating experimentally-observed solvent effects on reaction equilibria with the solvent polarity scale $E_{\mathrm{T}}(30)$. However, as these correlations are empirical and different for every reaction, they cannot be applied to predict the influence of solvents on other reactions than the ones investigated.

Solvent effects on the kinetics of hydroformylation (HYFO) reactions have already been studied experimentally in conventional organic solvents, in supercritical carbon dioxide, and in ionic liquids. ${ }^{2-7}$ It was shown that the use of different solvents can affect both, the reaction rate and the selectivity for the desired $n$-aldehyde. There is also consensus, that besides affecting the HYFO reaction, solvents also affect the solubility of the synthesis gas in the reaction mixture. However, in most works the gas solubilities were not quantified which prevented a profound analysis of the observed solvent effects.

Deshpande et al. ${ }^{8}$ used different alcohols as solvents for the HYFO of allyl alcohol and observed an increasing selectivity as well as increasing reaction rate with increasing chain length of the solvent alcohols. They stated that the gas solubility only marginally depends on the chain length of the solvent alcohols and attributed the observed effects to interactions of the solvent alcohols with the catalyst and the allyl alcohol. In more detailed works ${ }^{9-11}$, the effect of solvents and additives on HYFO kinetics was ascribed to shifted equilibria between different catalytic species that were experimentally observed. Reaction mechanisms of the biphasic HYFO of longchain substrates using different catalysts were reviewed by De et al. ${ }^{12}$. The reaction network of the HYFO has been investigated by Markert et al. and Kiedorf et al. ${ }^{13,14}$ 
In this work we consider the solvent effect on the HYFO of 1-dodecene to $n$-tridecanal (framed reaction in Figure 1), which is part of a reaction network that also comprises side reactions leading to the formation of side products such as iso-aldehydes, iso-dodecenes and dodecane.

Quantum-chemical (QC) calculations offer the possibility to obtain thermodynamic properties such as standard reaction enthalpies and entropies as contributions to the standard Gibbs energy of reaction. The HYFO reaction mechanism has been in the focus of many QC investigations which were recently reviewed by Kégl ${ }^{15}$. While the HYFO mechanism has been extensively studied by computational means, the thermodynamic reaction equilibrium has received much less attention.

Comparison of computational results with experiments usually relies on calculated standard reaction enthalpies in the gas phase with reference to experimental enthalpies of formation for the olefin and aldehyde. Rush et al. ${ }^{16}$ used highly advanced explicitly-correlated coupled-cluster $\operatorname{CCSD}(\mathrm{T})-\mathrm{F} 12$ calculations and obtained an enthalpy of reaction of the propene HYFO in excellent agreement with the experiment (within $2 \mathrm{~kJ} \mathrm{~mol}^{-1}$ ). Decker et al. ${ }^{17}$ used Møller-Plesset second-order perturbation theory (MP2) calculations for the ethylene HYFO with a deviation of $15 \mathrm{~kJ} \mathrm{~mol}^{-1}$ from the experiment.

To the best of our knowledge, there are no experimental data nor thermodynamic modeling for solvent effects on a HYFO reaction equilibrium available in the literature. In this work, we therefore investigate the solvent effect on the reaction equilibrium of the 1-dodecene HYFO by means of both, experiments and thermodynamic modeling. A two-component solvent system composed of decane and $N, N$-dimethylformamide (DMF) as introduced by Behr et al. ${ }^{2}$ was applied for this purpose. We investigated the effect of different decane/DMF ratios on the HYFO 
reaction equilibrium at temperatures between $368 \mathrm{~K}$ and $388 \mathrm{~K}$. The reaction was performed using a $\mathrm{Rh}(\mathrm{CO})_{2}(\mathrm{acac})$ precatalyst and a BIPHEPHOS ligand.

Below we present QC calculated standard reaction enthalpies and Gibbs energies (thermodynamic equilibrium constants) for the uncatalyzed reaction. A high level of electron correlation is required to obtain results of chemical accuracy (within $4 \mathrm{~kJ} \mathrm{~mol}^{-1}$ ). Solvent effects were treated using an implicit solvation $\operatorname{model}^{18}$ to estimate the effect of solvents on the standard Gibbs energy of reaction at infinite dilution in liquid solvents.

Using the QC determined thermodynamic equilibrium constant, equilibrium concentrations of reactants and products at varying solvent compositions and temperatures were predicted using fugacity coefficients obtained from the Perturbed-Chain Statistical Associating Fluid Theory ${ }^{19}$ (PC-SAFT). PC-SAFT was already successfully applied to simultaneously describe phase equilibria and reaction equilibria e.g. those involved in the carbon dioxide absorption in aqueous amine solutions ${ }^{20}$. Moreover, PC-SAFT has been shown earlier to be able to predict the solvent effect on esterification reaction equilibria in very good agreement with experimental data ${ }^{21}$.

To validate the predictions, the equilibrium concentrations of the liquid reactants/products were finally determined experimentally, exploiting the concentrations of $\mathrm{CO}$ and $\mathrm{H}_{2}$ in the reaction mixture as predicted by PC-SAFT.

\section{THEORY}

Thermodynamic description of gas solubilities and reaction equilibria. Homogeneouslycatalyzed HYFO reactions take place in the liquid phase. The gaseous reactants $\mathrm{CO}$ and $\mathrm{H}_{2}$ are dissolved in the liquid reaction mixture according to their gas solubility at reaction pressure and 
temperature. Therefore, gas solubilities and reaction equilibrium need to be described simultaneously.

For a system consisting of $\mathrm{C}$ components in a liquid phase (l) and a gas phase (g), the following phase equilibrium conditions hold:

$$
x_{i}^{(l)} \cdot \varphi_{i}^{(l)}=x_{i}^{(g)} \cdot \varphi_{i}^{(g)} \quad i=1, \ldots C
$$

Thereby $x_{i}$ and $\varphi_{i}$ denote the mole fraction and the fugacity coefficient of a component $i$, respectively. For $i=\mathrm{CO}$ or $\mathrm{H}_{2}, x_{i}^{(l)}$ corresponds to the solubility of these gases in the liquid reaction mixture.

Reaction equilibria can be characterized by the standard Gibbs energy of reaction $\Delta^{R} g^{0}$ referring to the ideal-gas standard state at the reference pressure $p^{0}=1.013$ bar. $\Delta^{R} g^{0}$ is defined according to

$$
\Delta^{R} g^{0}\left(T, p^{0}\right)=\Delta^{R} h^{0}\left(T, p^{0}\right)-T \cdot \Delta^{R} S^{0}\left(T, p^{0}\right)
$$

where $\Delta^{R} h^{0}$ is the standard enthalpy of reaction and $\Delta^{R} S^{0}$ is the standard entropy of reaction. The standard reaction properties $\Delta^{R} g^{0}, \Delta^{R} h^{0}$ and $\Delta^{R} S^{0}$ can be obtained from the respective standard properties of formation - the standard Gibbs energies of formation $\Delta^{F} g_{i}^{0}$, the standard enthalpies of formation $\Delta^{F} h_{i}^{0}$ and the standard entropies of formation $\Delta^{F} S_{i}^{0}$ of the reactants/products:

$$
\Delta^{R} g / h / s^{0} T, p^{0}={ }_{i} v_{i} \cdot \Delta^{F} g / h / s_{i}^{0} T, p^{0}
$$

where $v_{i}$ denotes the stoichiometric coefficient of component $i$ in the respective reaction. $\Delta^{R} g^{0}$ is related to the thermodynamic equilibrium constant $K_{f}$ via

$$
\Delta^{R} g^{0} T, p^{0}=-R T \cdot \ln K_{f}\left(T, p^{0}\right)
$$


where $R$ is the universal gas constant. $\Delta^{R} g^{0}$ refers to the ideal-gas standard state at the reference pressure $p^{0}=1.013$ bar. $K_{f}$ is based on the fugacities of reactants and products and is defined according to:

$$
K_{f}=i_{i} x_{i}^{(l)} v_{i} \cdot i_{i}^{(l)}{ }^{v_{i}} \cdot{\frac{p}{p^{0}}}^{v_{i}}
$$

For the HYFO reaction investigated in this work (see Figure 1), $K_{f}$ results in:

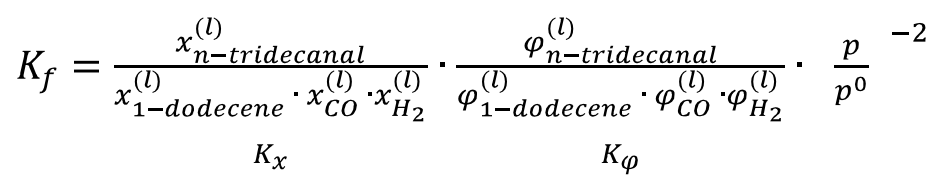

The thermodynamic equilibrium constant $K_{f}$ only depends on temperature. The temperature dependence of $K_{f}$ can be described using $\Delta^{R} h^{0}$ according to

$\ln \frac{K_{f, 1}}{K_{f, 2}}=-\frac{\Delta^{R} h^{0}}{R} \cdot \frac{1}{T_{1}}-\frac{1}{T_{2}}$

$K_{f}$ does neither depend on pressure nor on the solvents used as reaction medium. In contrast to this, the fugacity coefficients and therewith the mole fractions at reaction equilibrium depend on the amounts of all components in the reaction mixture including the solvent. Thus, once $K_{f}$ is known, the mole fractions of reactants and products at reaction equilibrium in different solvents can be predicted using the fugacity coefficients of these components (Eq. 6). As the gas solubilities $x_{C O}^{(l)}$ and $x_{H_{2}}^{(l)}$ not only depend on the solvent but also on the conversion of 1 dodecene to $n$-tridecanal, the conditions for phase equilibrium (Eq. 1) and reaction equilibrium (Eq. 6) have to be solved simultaneously for predicting the equilibrium concentrations of all reactants/products.

Besides the ideal-gas standard state, also the standard state of infinite dilution in a liquid solvent can be considered. For that purpose, Eq. 4 can be re-written as

$$
\Delta^{R} g^{\infty} T, p, \text { solvent }=-R T \cdot \ln \left(K_{a}^{\infty}(T, p, \text { solvent })\right)
$$


In contrast to $\Delta^{R} g^{0}, \Delta^{R} g^{\infty}$ does depend on the solvent and on the pressure $p$. The thermodynamic equilibrium constant $K_{a}^{\infty}$ for the infinite-dilution standard state is defined as

$$
K_{a}^{\infty}=K_{x} \cdot K_{\varphi} / K_{\varphi}^{\infty}
$$

with $K_{\varphi}^{\infty}$ being calculated from the fugacity coefficients of the reactants/products at infinite dilution in the liquid solvent. The Gibbs energies of reaction at the two different standard states are related according to

$$
\Delta^{R} g^{\infty} T, p=\Delta^{R} g^{0} T, p^{0}+R T \cdot \ln \frac{p}{p^{0}}+R T \cdot \ln \left(K_{\varphi}^{\infty}\right)
$$

PC-SAFT. In this work, PC-SAFT ${ }^{19}$ was applied for calculating the gas solubilities, $x_{C O}^{(l)}$ and $x_{H_{2}}^{(l)}$, as well as the fugacity coefficients of reactants and products in the reaction mixture, $\varphi_{i}^{(l)}$. PC-SAFT is based on the perturbation theory of Barker and Henderson ${ }^{22}$ and considers the residual Helmholtz energy of a system $A^{\text {res }}$ as a sum of various contributions:

$$
A^{\text {res }}=A^{h c}+A^{\text {disp }}+A^{\text {assoc }}+A^{\text {dipole }}
$$

Thereby the hard-chain (hc) reference system represents the repulsive interactions between the molecules. The attractive interactions, such as the dispersion (disp), association (assoc) and polar (dipole) interactions, are described as perturbations of the reference system. In this work, the association contribution was set to zero as no associating components were considered. A detailed description of the PC-SAFT model can be found in the literature ${ }^{19,23}$.

PC-SAFT treats a molecule as a chain of spherical segments. Pure components are characterized by three parameters, namely the segment number $m_{i}$, the segment diameter $\sigma_{i}$ and the dispersion-energy parameter $u_{i} / k_{B}$. Modeling of polar components also requires the dipole moment $\mu_{i}$. The pure-component parameters are usually identified by fitting them to vapor pressures and liquid densities of the respective components. 
For the description of mixtures, Berthelot-Lorenz mixing rules are applied for the segment diameter and the dispersion energy

$$
\begin{aligned}
& \sigma_{i j}=\frac{\sigma_{i}+\sigma_{j}}{2} \\
& u_{i j}=1-k_{i j} \quad \overline{u_{i} \cdot u_{j}}
\end{aligned}
$$

where $k_{i j}$ is a binary parameter used for adjusting the dispersion energy between unlike components. It is usually fitted to binary phase-equilibrium data. In some cases, the $k_{i j}$ parameter is treated as temperature-dependent according to:

$$
k_{i j}=a \cdot T+b
$$

The fugacity coefficients required in Eq. (1) and (6) are calculated from the residual Helmholtz energy by

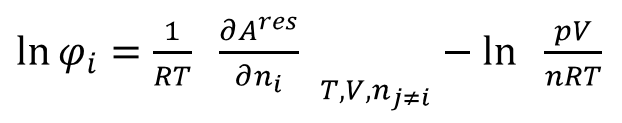

where $n_{i}$ is the mole number of component i, $n$ the total mole number and $V$ the volume of the system.

PC-SAFT parameters were available from literature for all components considered in this work and are presented in the supplementary material (section S1).

Quantum-chemical prediction of thermochemical data. The standard Gibbs energy of reaction can also be obtained from QC calculations using Eq. (2). QC calculations based on the electronic Schrödinger equation yield the energy of reaction at $0 \mathrm{~K}$ in the gas phase $\Delta^{R} e^{\text {gas }}$ to which the nuclear repulsion term, zero-point vibrational energy (ZPVE) and corrections from statistical mechanics are applied (such as the rigid rotor and harmonic oscillator approximations; a detailed introduction can be found in the literature ${ }^{24}$ ). 
When investigating the effect of solvation, the solute-solvent interaction must be accounted for. The polar solvation Gibbs energy $\Delta^{R} g^{s o l}$, which is the electrostatic interaction between the solute and the solvent (treated as a continuum dielectric medium), was computed using the COSMO solvation model ${ }^{25}$. The COSMO solvation model is an implicit solvation model, which uses the scaled conductor boundary condition as a robust approximation to the exact dielectric equations and reduces the outlying charge errors as compared to other implicit solvation models.

The Gibbs energy of reaction in the standard state of infinite dilution in a liquid solvent is then obtained based on the following equation

$$
\Delta^{R} g^{\infty}=\Delta^{R} e^{g a s}+\Delta^{R} g_{R R H O, l}^{0}+\Delta^{R} g^{s o l}
$$

where $\Delta^{R} g_{R R H O, l}^{0}$ is the change in translational, rotational and vibrational free energy computed using the rigid-rotor and harmonic oscillator approximation for the liquid state, $\Delta^{R} g^{s o l}$ is the change in solvation energy computed using a polarizable continuum model, here COSMO.

QC calculations refer to an ideal-gas state at a pressure of 1 bar. When going from the idealgas state $\left(\Delta^{R} g_{R R H O}^{0}\right)$ to the infinitely-diluted liquid state $\left(\Delta^{R} g_{R R H O, l}^{0}\right)$, the change in standard state must be accounted for leading to

$$
\Delta^{R} g_{R R H O, l}^{0}(T, p)=\Delta^{R} g_{R R H O}^{0}(T, p)-R T \cdot \ln \frac{1}{v}
$$

where $v$ is the molar volume of an ideal gas in $\mathrm{L} / \mathrm{mol}$ at the specified pressure and temperature. For example, at $1 \mathrm{bar}$ and $298 \mathrm{~K}$ this contributes a correction of $7.95 \mathrm{~kJ} \mathrm{~mol}^{-1}$ and at $1 \mathrm{bar}$ and $378 \mathrm{~K}$ a correction of $10.83 \mathrm{~kJ} \mathrm{~mol}^{-1}$.

Zhou and Gilson ${ }^{26}$ have shown that the rigid rotor-harmonic oscillator approximation is also valid in solution since the molecule in solution still has the same degrees of freedom at infinite dilution and the same thermodynamic corrections as in the gas phase $\left(\Delta^{R} g_{R R H O}^{0}\right)$ are appropriate. 
Here, we present a comparison of different quantum chemical computational approaches to calculate the thermochemical data of the hydroformylation reaction. On the one hand, we used density functional theory (DFT) calculations which are already established for the calculation of homogeneously catalyzed reactions in the literature. On the other hand, we applied wavefunction-based methods with different levels of electron correlation that are not yet frequently used for those tasks. The QC calculation of thermochemical properties requires first geometry-optimized molecule structures. Structures of reactants and the product were fully optimized using different density functional theory (DFT) methods BP86 ${ }^{27,28}$ and B3LYP ${ }^{27,29}$ and ab initio Møller-Plesset second order perturbation theory (MP2) to include electron correlation ${ }^{30}$. The recently published DLPNO-CCSD(T) method employs pair-natural orbitals (PNOs) and domain-based techniques and shows a nearly linear scaling of the computation time with the system size $e^{31}$.

The localized correlated coupled cluster $\operatorname{CCSD}(\mathrm{T})$ calculations were single-point calculations on the optimized MP2/def2-TZVP geometries. For the atomic orbitals, a def2-TZVP ${ }^{32}$ basis set was used for all methods except for $\operatorname{CCSD}(\mathrm{T})$ for which a cc-pVXZ ${ }^{33,34}$ basis set was used. The MP2 perturbative calculations were all-electron calculations without any frozen core orbitals. For B3LYP empirical atomic dispersion corrections D3 from Grimme ${ }^{35}$ were taken into account. BP86, MP2 and $\operatorname{CCSD}(\mathrm{T})$ calculations were done in the resolution of the identity (RI) approximation $^{36}$.

Calculation of second derivatives were performed to consider thermodynamic corrections to the energies at 298 and $378 \mathrm{~K}$, respectively. For the coupled cluster energies, thermodynamic contributions were taken from MP2 calculations. The calculations were performed using 
Turbomole V6.4 ${ }^{37}$ or Orca v.3.0.2 $2^{38}$. Thus, thermochemical properties for the HYFO reaction of 1-dodecene were calculated at various levels of accuracy to critically assess their performance.

\section{EXPERIMENTS}

Materials. $N, N$-dimethylformamide (VWR, $99.99 \%$ ), decane (TCI, $99.5 \%$ ), 1-dodecene (Merck, $94.7 \%), \mathrm{Rh}(\mathrm{CO})_{2}(\mathrm{acac})$ (Umicore, $\left.40.0 \% \mathrm{Rh}\right), 6,6^{\prime}$-[(3,3'-Di-tert-butyl-5,5'-dimethoxy1,1'-biphenyl-2,2'-diyl)bis(oxy)]bis(dibenzo[d,f][1,3,2]dioxaphosphepin) (BIPHEPHOS, Molisa, $>99 \%)$, and synthesis gas $\left(\mathrm{CO} / \mathrm{H}_{2}=1: 1\right.$, Linde, $\left.\mathrm{CO}=(50.0 \pm 1) \%\right)$ were used as delivered without further purification. 1-dodecene (Fluka, $97.1 \%$ ), $n$-tridecanal (Sigma, $99.6 \%$ ), dodecane (Sigma Aldrich, $99.1 \%$ ), decane (TCI, $99.5 \%$ ), and DMF (VWR, $99.99 \%$ ) were used as calibration standards for the gas-chromatographic analysis.

Reaction equilibrium experiments. HYFO experiments were performed in a multiple reactor system (Parr Instrument Company) equipped with a $75 \mathrm{~mL}$ stainless steel reactor mounted with a PTFE coated gas cylinder, a magnetic stirrer, a heating jacket and a gas supply. The pressure was monitored using a pressure transducer $(\mathrm{G} 2$ Pressure Transducer, $\pm 1.00 \%$ total error band, Ashcroft Instruments $\mathrm{GmbH}$ ) and the temperature was monitored and regulated using a thermocouple (Type $\mathrm{J}, \mathrm{Fe}-\mathrm{CuNi}, \pm 1.5 \mathrm{~K}$ ) and a process controller (Parr Instrument Company). The experimental setup is illustrated in Figure 2.

In a typical experiment, the precatalyst $\mathrm{Rh}(\mathrm{CO})_{2}(\mathrm{acac})(0.05$ mol- $\%)$ and the ligand BIPHEPHOS (molar ratio Ligand/Rh $=3.3$ ) were dissolved in the solvents decane and DMF $\left(V_{\text {solvent }}=35.58 \mathrm{~mL}\right)$ with varying mass ratios $w_{\text {decane }} / w_{D M F}(\mathrm{wt}-\% / \mathrm{wt}-\%)$. This catalyst solution was evacuated (10 mbar) and pressurized (1 bar) with synthesis gas three times under stirring followed by a complete evacuation (10 mbar) prior to the catalyst pretreatment. The 
latter was started by pressurizing the reactor with synthesis gas (14 bar) and heating the catalyst solution to reaction temperature for $30 \mathrm{~min}$ at $1200 \mathrm{rpm}$. During the catalyst pretreatment the valve was closed and the PTFE-coated gas cylinder was filled with 1-dodecene $\left(V_{1-\text { dodecene }}=9.42\right.$ $\left.\mathrm{mL}, V_{\text {total }}=V_{\text {solvent }}+V_{1 \text {-dodecene }}=50 \mathrm{~mL}, c_{1 \text {-dodecene }}=0.89 \mathrm{~mol} \mathrm{~L}^{-1}\right)$ evacuated $(10 \mathrm{mbar})$ and pressurized with synthesis gas ( 1 bar) three times followed by a completing pressurization with synthesis gas (21 bar).

The experiment was started after the catalyst pretreatment by opening the valve between the reactor and the gas cylinder for $10 \mathrm{~s}$. Performing test measurements, the reaction was proven to be in the equilibrium state after the pressure remained constant for a duration of $30 \mathrm{~min}$. Equilibrium pressures between 0.3 and 1.1 bar were obtained. Finally, a sample of the reaction mixture at equilibrium was taken for every experiment and analyzed via gas chromatography.

HYFO reactions were conducted for three different decane/DMF ratios 80/20, 60/40 and 0/100 and at three different temperatures $T=368,378,388 \mathrm{~K}$. Every experiment was conducted three times and average values are reported.

For validation purposes, an additional experiment with a different catalyst concentration was conducted three times at $378 \mathrm{~K}$ and a decane/DMF ratio of $60 / 40$. The same concentrations as in the original experiment were obtained which shows that our results do not depend on the starting conditions and therefore the equilibrium state of the reaction was achieved.

Gas chromatographic analytics. Samples of the reaction mixtures at equilibrium $(0.5 \mathrm{~mL})$ were diluted with 2-propanol $(1.5 \mathrm{~mL})$ and analyzed using a gas chromatograph (GC, 6890 Series, Agilent) equipped with a flame ionization detector, a polar polyethylene glycol stationary phase (HP-INNOWax, 2x $60 \mathrm{~m}, \mathrm{~d}=250 \mu \mathrm{m}$, film $=0.5 \mu \mathrm{m}$, Agilent) and helium (Linde, $99.999 \%$ ) as mobile phase. The samples were analyzed according to the reaction network (Figure 1) for the 
mole fractions of 1-dodecene, dodecene isomers, $n$-tridecanal, aldehyde isomers and dodecane as well as for the solvents decane and DMF. Decane was used as internal standard. Due to the lack of commercially-available calibration standards, response factors of 1-dodecene and $n$-tridecanal were also used for dodecene isomers and aldehyde isomers, respectively. Every sample was analyzed three times for the above-mentioned analytes and a mean mole fraction was calculated. The relative standard deviation was found to be less than $1 \%$.

\section{EXPERIMENTAL RESULTS}

Determination of $\boldsymbol{K}_{x}$. Calculation of $K_{x}$ (Eq. 6) requires the equilibrium mole fractions of the reactants/products of the HYFO reaction. The equilibrium mole fractions of 1-dodecene and $n$ tridecanal, $x_{1-\text { dodecene }}^{(l)}$ and $x_{\text {tridecanal }}^{(l)}$ were obtained analytically by gas chromatography as described above. The equilibrium mole fractions of the gases $x_{C O}^{(l)}$ and $x_{H_{2}}^{(l)}$ were calculated by solving Eq. 1 using PC-SAFT based on the following information:

- the experimental temperature and equilibrium pressure

- the analytically-determined mole fractions of all liquid components $\left(x_{1-\text { dodecene }}^{(l)}\right.$, $\left.x_{\text {tridecanal }}^{(l)}, \quad x_{\text {decane }}^{(l)}, \quad x_{D M F}^{(l)}, \quad x_{\text {iso-dodecenes }}^{(l)}, \quad x_{\text {iso-aldehydes }}^{(l)}, \quad x_{\text {dodecane }}^{(l)}\right)$ at reaction equilibrium (all these components were accounted for when calculating the gas solubilities $x_{C O}^{(l)}$ and $\left.x_{H_{2}}^{(l)}\right)$

Two assumptions were made for this purpose. Firstly, it was assumed that $\mathrm{CO}$ and $\mathrm{H}_{2}$ were present in the gas phase in a 1:1 ratio $x_{C O}^{v}=x_{H_{2}}^{v}$. This assumption is reasonable as synthesis gas of a molar 1:1 ratio was used and only small amounts of this gas were dissolved in the liquid phase under the experimental conditions. To estimate the real composition of the gas phase, a 
mole balance for the gases in the two phases $\left(n_{C O}^{(l)}+n_{C O}^{(g)}=n_{H_{2}}^{(l)}+n_{H_{2}}^{(g)}\right)$ was combined with Eq. 1. The relative deviation of the real gas-phase composition from the assumed one $(1: 1)$ is smaller than $10 \%$ for both, $x_{C O}^{v}$ and $x_{H_{2}}^{v}$.

Secondly, dodecane and decane were both treated as being decane for calculating the gas solubilities with PC-SAFT. The resulting $K_{x}$ values are presented in Table 1 for temperatures ranging from $368 \mathrm{~K}$ to $388 \mathrm{~K}$ and for compositions of the solvent system $w_{\text {decane }} / w_{D M F}$ ranging from $80 / 20$ to $0 / 100$.

Due to the very low equilibrium pressures $(0.3-1.1$ bar $)$, the mole fractions of the gases in the liquid reaction mixture at equilibrium are very low (in the order of $10^{-4}$ ). Moreover, due to almost full conversion at equilibrium, the concentration of 1-dodecene is very low compared to the one of $n$-tridecanal. These concentrations cause large $K_{x}$ values (in the order of $10^{8}$ to $10^{10}$ ).

Solvent effect on $\boldsymbol{K}_{\boldsymbol{x}}$. At all investigated temperatures, $K_{x}$ increases with an increasing content of the polar solvent DMF in the solvent mixture. In the $80 / 20$ decane/DMF mixture, the equilibrium gas mole fractions $x_{C O}^{(l)}$ and $x_{H_{2}}^{(l)}$ are approximately four times higher than in pure DMF whereas the mole-fraction ratio of $n$-tridecanal and 1-dodecene $x_{n \text {-tridecanal }} / x_{1-\text { dodecene }}$ does not significantly depend on the DMF content in the solvent system. Therefore, the solvent effect on $K_{x}$ is mainly ascribed to the different gas solubilities $\left(x_{C O}^{(l)}\right.$ and $\left.x_{H_{2}}^{(l)}\right)$ in the decane/DMF mixtures. Thus, depending on the temperature, $K_{x}$ is 13 to 24 times higher in pure DMF, than in the $80 / 20$ decane/DMF-mixture.

Temperature effect on $\boldsymbol{K}_{x}$. At all compositions of the solvent system $w_{\text {decane }} / w_{D M F}, K_{x}$ decreases with increasing temperature. The main reason for this observation are again the changes in the gas solubilities of $\mathrm{CO}$ and $\mathrm{H}_{2}$. The gas solubilities increase with increasing 
temperature as already shown by Vogelpohl et al. ${ }^{39,40}$. The increase of the gas mole fractions $x_{C O}$ and $x_{\mathrm{H}_{2}}$ with increasing reaction temperature in Table 1 is even more pronounced as also the equilibrium pressure increases at the same time. Thus, depending on the composition of the solvent system $K_{x}$ at $368 \mathrm{~K}$ is 8 to 11 times higher than the one at $388 \mathrm{~K}$.

\section{DISCUSSION}

\section{Determination of the reaction enthalpy $\Delta^{R} h^{0}$ and Gibbs energy of reaction $\Delta^{R} g^{0}$ at the}

ideal-gas standard state. The reaction enthalpy $\Delta^{R} h^{0}$ and Gibbs energy of reaction $\Delta^{R} g^{0}$ at the ideal-gas standard state are of particular interest for the thermodynamic characterization of the reaction equilibrium of the 1-dodecene HYFO. Moreover, these properties build the basis for the calculation of the standard Gibbs energy of reaction $\Delta^{R} g^{\infty}$ at infinite dilution in liquid solvents (Eq. 10) and the thermodynamic equilibrium constant $K_{f}$ (Eqs. 4 and 7). In this work, two approaches were applied to obtain $\Delta^{R} h^{0}$ and $\Delta^{R} g$.

Firstly, we applied standard enthalpies of formation $\Delta^{F} h_{i}^{0}$ and standard Gibbs energies of formation $\Delta^{F} g_{i}^{0}$ of the reactants/products (Eq. 3) taken from literature. ${ }^{41-44}$ The results are summarized in Table 2. As discussed in the supplementary material (section S2), the uncertainties of this method were estimated to $u \Delta^{R} h^{0}= \pm 5.49 \mathrm{~kJ} / \mathrm{mol}$ and $u \Delta^{R} g^{0}= \pm 5.65$ $\mathrm{kJ} / \mathrm{mol}$.

Secondly, the standard reaction enthalpy $\Delta^{R} h^{0}$ and the standard Gibbs energy of reaction $\Delta^{R} g^{0}$ were determined via QC calculations. A QC prediction of thermochemical data is still a challenge for theoretical approaches in terms of accuracy. Density functional theory (DFT) is a computationally-efficient approach to treat electron correlation effects. However, results obtained from DFT calculation show a large, non-systematic deviation from experimental data 
and are therefore not used for calculations of thermochemical properties in this work. An overview of the $\Delta^{R} h^{0}$ and $\Delta^{R} g^{0}$ values obtained from different QC methods is provided in the supplementary material (section S3).

The calculations using MP2 and coupled-cluster methods were consistent and yield reliable reaction energies compliant with each other (see supplementary material, Table S4). With a moderate basis set (def2-TZVP), MP2 gives a reaction energy of $-157.52 \mathrm{~kJ} \mathrm{~mol}^{-1}$, which yields a standard reaction enthalpy of $-123 \mathrm{~kJ} \mathrm{~mol}^{-1}$ and standard Gibbs energy of reaction of $-17.86 \mathrm{~kJ}$ $\mathrm{mol}^{-1}$ at $378 \mathrm{~K}$. Coupled-cluster calculations with Dunning's correlation-consistent basis sets (CC-pVnZ, n=2, 3, 4) were performed in order to benchmark the accuracy of MP2 (see supplementary material, Table S4). The results demonstrate the slow but systematic convergence of the reaction energy with basis set size and allows to use a well-defined extrapolation to the complete basis set (CBS) limit. A three-parameter CBS extrapolation scheme

$$
Y x=Y_{C B S}+A e^{-B x} \text { for } \mathrm{x}=2,3,4 \text {. }
$$

was used to obtain a valid benchmark of $-154.7 \mathrm{~kJ} \mathrm{~mol}^{-1}$ which agrees well with the MP2 results $\left(-157.5 \mathrm{~kJ} \mathrm{~mol}^{-1}\right)$. Subsequently, results from MP2 calculations for which second derivatives are available, were used consistently. With thermodynamic corrections from MP2 calculations, a reliable estimate of the standard reaction enthalpy $\Delta^{R} h^{0}$ of $-118.6 \mathrm{~kJ} \mathrm{~mol}^{-1}$ and standard Gibbs energy of reaction $\Delta^{R} g^{0}$ of $-37.1 \mathrm{~kJ} \mathrm{~mol}^{-1}$ at $298 \mathrm{~K}$ and 1 bar was obtained.

The uncertainty of both, $\Delta^{R} h^{0}$ and $\Delta^{R} g^{0}$ obtained from MP2 calculations was estimated to be \pm $4.00 \mathrm{~kJ} / \mathrm{mol}$ ('chemical accuracy'). Values and uncertainties of $\Delta^{R} h^{0}$ and $\Delta^{R} g^{0}$ obtained with MP2 are also presented in Table 2.

As can be seen, the values obtained with the two different methods agree well within their uncertainty limits. Moreover, the temperature dependence of the standard reaction enthalpy $\Delta^{R} h^{0}$ 
is quite small and both methods show a small decrease of $\Delta^{R} h^{0}$ with increasing temperature (see supplementary material (section S4) for a calculated temperature dependence of $\Delta^{R} h^{0}$ and $\left.T \cdot \Delta^{R} S^{0}\right)$. Both, the values for the standard reaction enthalpy $\Delta^{R} h^{0}$ as well as the ones for the standard Gibbs energy of reaction $\Delta^{R} g^{0}$ obtained from MP2 show a smaller uncertainty than those obtained from the formation properties $\Delta^{F} h_{i}^{0}$ and $\Delta^{F} g_{i}^{0}$. Therefore, the MP2 values were used for further calculations in this work.

\section{Qualitative prediction of solvent effect on $K_{x}$ based on the standard Gibbs energy of} reaction $\Delta^{R} g^{\infty}$ at infinite dilution in liquid solvents. In this work two methods for the prediction of the solvent effect on $K_{x}$ are presented - firstly a qualitative prediction and secondly a quantitative prediction, whereas the qualitative prediction is based on the standard Gibbs energy of reaction $\Delta^{R} g^{\infty}$ at infinite dilution in liquid solvents.

Assuming that the thermodynamic behavior of the reactants/products in the reaction mixture would be the same as at infinite dilution, the ratio $K_{\varphi} / K_{\varphi}^{\infty}$ in Eq. 9 becomes one, which results in a direct relation between $\Delta^{R} g^{\infty}$ and $K_{x}$ (Eq. 8). Two different ways were applied to determine $\Delta^{R} g^{\infty}$ in a decane/DMF-mixture $\left(w_{\text {decane }} / w_{D M F}=80 / 20\right)$ as well as in pure DMF ( $w_{\text {decane }} /$ $w_{D M F}=0 / 100$ ). Both ways are based on the standard Gibbs energy of reaction $\Delta^{R} g^{0}$ at the idealgas standard state at $378 \mathrm{~K}$ calculated using MP2 (Table 2).

Firstly, the Gibbs energy of reaction $\Delta^{R} g^{\infty}$ at infinite dilution in liquid solvents was obtained from QC calculations. In all QC calculations performed in this work, the predicted solvent effect was almost independent of the method used and added another 4-5 and 11-12 $\mathrm{kJ} \mathrm{mol}^{-1}$ to the standard Gibbs energy of reaction in pure decane and DMF, respectively, as shown in supplementary material (section S5). Performing COSMO calculations at $w_{\text {decane }} / w_{D M F}$ ratios of $80 / 20$ and $0 / 100$ and correcting for the change in standard state $\left(11.78 \mathrm{~kJ} \mathrm{~mol}^{-1}\right.$ at $0.74 \mathrm{bar}$ and 
$378 \mathrm{~K}$; and $12.60 \mathrm{~kJ} \mathrm{~mol}^{-1}$ at 0.57 bar and $378 \mathrm{~K}$, respectively) one obtains the Gibbs energy of reaction $\Delta^{R} g^{\infty}$ at infinite dilution in liquid solvents. These values are included in Table 3 as "MP2/COSMO".

Secondly, the standard Gibbs energy of reaction $\Delta^{R} g^{\infty}$ at infinite dilution in liquid solvents was also calculated according to Eq. 10 using the fugacity coefficients at infinite dilution calculated from PC-SAFT. The so-obtained standard Gibbs energies of reaction $\Delta^{R} g^{\infty}$ at infinite dilution in liquid solvents are also presented in Table 3 denoted as "MP2/PC-SAFT".

As it can be seen, the values obtained from the two methods agree qualitatively but differ in absolute values and also regarding the strength of the solvent effect. While PC-SAFT explicitly accounts for binary interactions among the solvents and the reacting species via the binary $k_{i j}$ parameters (Eq. 13) fitted to binary phase-equilibrium data, COSMO is an implicit solvation model in which the reacting species are embedded in a dielectric continuum surrounding the molecular cavity. COSMO, in contrast to PC-SAFT, does not explicitly include solvent molecules. Nevertheless, the standard Gibbs energies of reaction $\Delta^{R} g^{\infty}$ at infinite dilution decrease with increasing DMF content for both MP2/COSMO and MP2/PC-SAFT, which leads to increasing $K_{x}$-values (Eq. 8). This is in qualitative agreement with the experimental observations (Table 1).

This shows that the solvent effect on the reaction equilibria, i.e. on $K_{x}$, can be predicted qualitatively via QC calculations alone as well as via a combination of QC calculations and PCSAFT without using any experimental reaction data.

Determination of the thermodynamic equilibrium constant $\boldsymbol{K}_{\boldsymbol{f}}$. The quantitative prediction of the solvent effect on $K_{x}$ via Eq. 6 requires the thermodynamic equilibrium constant $K_{f} . K_{f}$ 
was calculated at different temperatures via Eqs. 4 and 7 using the reaction enthalpy $\Delta^{R} h^{0}$ and the Gibbs energy of reaction $\Delta^{R} g^{0}$ at the ideal-gas standard state presented in Table 2.

The standard Gibbs energy of reaction $\Delta^{R} g^{0}$ was used to determine the thermodynamic equilibrium constant $K_{f}$ at $378 \mathrm{~K}$ via Eq. 4 . Based on this, the $K_{f}$-values at $368 \mathrm{~K}$ and at $388 \mathrm{~K}$ were determined via Eq. 7 using the standard reaction enthalpy $\Delta^{R} h^{0}$ at $373 \mathrm{~K}$ and $383 \mathrm{~K}$, respectively.

For comparison, experimental thermodynamic equilibrium constants $K_{f, \exp }$ were calculated according to Eq. 6 using the experimentally-determined mole fractions of the HYFO reactants/products at the solvent composition $60 / 40\left(w_{\text {decane }} / w_{D M F}\right)$ and the respective fugacity coefficients obtained from PC-SAFT. The resulting two sets of values for the thermodynamic equilibrium constant from both, MP2 $\left(K_{f}\right)$ and experimental data combined with PC-SAFT $\left(K_{f, \exp }\right)$ are presented in Table 4.

As it can be seen in Table 4, the values obtained from MP2 calculations and from experimental data/PC-SAFT are in very good agreement, particularly keeping in mind the complexity of the reaction system and that MP2 is purely predictive. Further, it needs to be mentioned that the values for the thermodynamic equilibrium constant $K_{f}$ are about seven(!) orders of magnitude smaller than the $K_{x}$ values presented in Table 1 . This shows the very important role of the fugacity coefficients (Eq. 6) that capture the real thermodynamic behavior of the components and cannot be neglected at all. Neglecting the fugacity coefficients in (Eq. 6) would thus result in $K_{x}$ values in the order of $10^{2}$ to $10^{3}$ meaning that almost no $n$-tridecanal is formed which clearly contradicts the experimental results (Table 1). 
Moreover, the solvent effect observed could not have been described at all neglecting the fugacity coefficients, as these are the only physical properties that depend on the solvent and therewith enforce the change in $K_{x}$ via Eq. 6 .

\section{Quantitative prediction of the effects of temperature and solvent on $K_{x}$ based on the} thermodynamic equilibrium constant $\boldsymbol{K}_{\boldsymbol{f}}$. Using the values for the thermodynamic equilibrium constant $K_{f}$ determined via the standard reaction enthalpy $\Delta^{R} h^{0}$ and the standard Gibbs energy of reaction $\Delta^{R} g^{0}$ from MP2 calculations (Table 4), the solvent effect on $K_{x}$ was predicted with PCSAFT. The predictions were performed by simultaneously solving the conditions for the phase equilibrium (Eq. 1) and the reaction equilibrium (Eq. 6).

Thereby the side reactions of 1-dodecene to dodecane and to iso-aldehydes were neglected, which is a reasonable simplification as the side products were indeed formed to very small amounts only $\left(x_{\text {iso-aldehydes }}<0.25 \mathrm{~mol} \%, x_{\text {dodecane }}<0.50 \mathrm{~mol} \%\right)$. Furthermore, the fugacity coefficients of the reacting species of the main reaction (Eq. 6) mainly depend on the pressure and the composition of the solvent system. They are not significantly affected by the side reactions. The 1-dodecene isomerization was taken into account by setting the amount of 1dodecene among all olefin isomers to $1.3 \%$, which corresponds to the experimentally-obtained value. For the composition of the gas phase it was assumed that $\mathrm{CO}$ and $\mathrm{H}_{2}$ are present in a 1:1 ratio $x_{C O}^{v}=x_{H_{2}}^{v}$ as already stated in the section "Determination of $K_{x}$ ".

PC-SAFT requires the following information as input for the prediction of $K_{x}$ :

- the experimental temperature and equilibrium pressure

- the thermodynamic equilibrium constant $K_{f}$

- the starting concentrations used in the experiments $\left(x_{1-\text { dodecene }, 0}^{(l)}, x_{\text {decane, },}^{(l)}, x_{D M F, 0}^{(l)}\right)$ 
Based on that, PC-SAFT provides:

- the equilibrium concentrations of the reactants and products of the 1-dodecene HYFO $\left(x_{C O}^{l}, x_{H_{2}}^{l}, x_{1-\text { dodecene }}^{(l)}, x_{n-\text { tridecanal }}^{(l)}\right)$

- the fugacity coefficients of the reactants and products of the 1-dodecene HYFO $\left(\varphi_{C O}^{l}, \varphi_{\mathrm{H}_{2}}^{l}\right.$, $\left.\varphi_{1-\text { dodecene }}^{(l)}, \varphi_{n-\text { tridecanal }}^{(l)}\right)$

As the thermodynamic equilibrium constant $K_{f}$ is constant at a given temperature, the solvent influence on $K_{x}$ is completely determined by the solvent influence on the fugacity coefficients of the reactants and products of the HYFO. Table 5 summarizes the results of the PC-SAFT predictions for the equilibrium at temperatures ranging from $368 \mathrm{~K}$ to $388 \mathrm{~K}$ and for compositions of the solvent system $w_{\text {decane }} / w_{D M F}$ ranging from $80 / 20$ to $0 / 100$. These predictions were performed by simultaneously solving the conditions for the phase equilibrium (Eq. 1) and the reaction equilibrium (Eq. 6).

The fugacity coefficients of the liquid components 1 -dodecene and $n$-tridecanal are very small (in the order of $10^{-2}$ ) as their pure-component boiling points lie below the considered temperature range. They increase with increasing temperature. The fugacity coefficients of the gases in the liquid phase exceed the fugacity coefficients of 1 -dodecene and $n$-tridecanal by about four orders of magnitude. While the fugacity coefficients of the liquid components cancel more or less out when calculating $K_{\varphi}$ (Eq. 6), the fugacity coefficients of the gases do not. This is the main reason for the tremendous value of $K_{\varphi}$ (in the order of $10^{-8}$ ).

As to be seen from Table 5, $K_{\varphi}$ decreases with increasing DMF content in the solvent mixture. Although the fugacitiy coefficients of the liquid components 1-dodecene and $n$-tridecanal strongly depend on the composition of the solvent system, their fugacity coefficients are affected 
by the solvent system in a similar way and nearly cancel out (Eq. 6). The main reason for the strong influence of the solvent system composition on $K_{\varphi}$ is thus the influence on the fugacity coefficients of the gases $\mathrm{CO}$ and $\mathrm{H}_{2}$ as they strongly depend on the composition of the solvent system and do not cancel out in Eq. 6 .

Figure 3 shows the results for the PC-SAFT-predicted $K_{x}$ values (Table 5) in comparison with the experimental values presented in Table 1 . As can be seen, the predictions for $K_{x}$ are in very good agreement with the experimental data and cover both, the solvent effect as well as the temperature effect correctly with an overall ARD of $35.26 \%$. It is worth mentioning that this prediction is based on QC calculated thermodynamic equilibrium constants $K_{f}$ in the order of $10^{2}$ to $10^{3}$ (Table 4) aiming to describe experimentally-verified equilibrium compositions $\left(K_{x}\right)$ in the order of $10^{9}$ to $10^{10}$. The ratio of these two quantities is $K_{\varphi}$ (Eq. 6), which was purely predicted (PC-SAFT). Keeping this in mind, the agreement between predicted and experimentallydetermined $K_{x}$ values is quite remarkable.

An increase in $K_{x}$ with increasing DMF content in the solvent system was found by both, experiments and PC-SAFT predictions. Since $K_{\varphi}$ decreases with increasing DMF-content in the solvent mixture (Table 5), $K_{x}$ increases at the same time.

Comparing the experimental data (Table 1) and the predicted values (Table 5) for $K_{x}$, it can be seen that not only the predicted $K_{x}$ values but also the mole fractions of the reactants and the product are in very good agreement with the experimental data. The equilibrium concentrations of the gases $x_{C O}$ and $x_{H_{2}}$ were predicted highly accurate with an overall ARD of $0.95 \%$ and 1.05 $\%$, respectively. The mole fractions of 1-dodecene and $n$-tridecanal were predicted with an ARD of $25.31 \%$ and $25.02 \%$, respectively. This also means that the predictions correctly cover the 
experimental finding that the HYFO reaction equilibrium almost completely lies on the side of the reaction product $n$-tridecanal.

The temperature effect on $K_{x}$ is related to the temperature dependence of the thermodynamic equilibrium constant $K_{f}$ which is determined by the standard enthalpy of reaction $\Delta^{R} h^{0}$ according to Eq. 6. Standard enthalpies of reaction obtained from QC calculations as shown in Table 2 are $-123.14 \mathrm{~kJ} / \mathrm{mol}$ at $373 \mathrm{~K}$ and -123.33 at $383 \mathrm{~K}$. This is in very good agreement with the values for standard enthalpy of reaction $\Delta^{R} h^{0}$ obtained via applying Eq. 7 to the

experimentally-determined thermodynamic equilibrium constants $K_{f, \exp }$ shown in Table 4 ($127.72 \mathrm{~kJ} / \mathrm{mol}$ at $373 \mathrm{~K}$ and -111.63 at $383 \mathrm{~K})$.

\section{CONCLUSION}

With this work, we provided a consistent experimental investigation and thermodynamic description of the reaction equilibrium of the 1-dodecene HYFO. Reaction experiments were performed at temperatures between $368 \mathrm{~K}$ and $388 \mathrm{~K}$ at varying compositions of the applied decane/DMF-solvent system. Equilibrium compositions $\left(K_{x}\right)$ were determined by analyzing the mole fractions of the liquid components at reaction equilibrium and calculating the corresponding solubilities of the synthesis-gas components with PC-SAFT. It was found that $K_{x}$ strongly depends on the composition of the solvent system decane/DMF and increases with increasing DMF content as well as with decreasing temperature.

The QC calculation of thermochemical data poses a challenge in terms of accuracy. Standard DFT functionals are not appropriate to obtain accurate data. Moderate (MP2) to high levels (CC) of inclusion of electron correlation are needed to provide reliable results. Via QC calculations (MP2), the standard reaction enthalpy $\Delta^{R} h^{0}$ and the standard Gibbs energy of reaction $\Delta^{R} g^{0}$ 
were obtained allowing the calculation of the thermodynamic equilibrium constant $K_{f}$ at temperatures of 368,378 , and $388 \mathrm{~K}$. The so-obtained $K_{f}$ was found to be seven orders of magnitude lower than the experimentally-determined $K_{x}$. This huge discrepancy could be ascribed to the strong non-ideality expressed by the fugacity coefficients of the reactants and the product.

Using fugacity coefficients predicted from PC-SAFT, $K_{x}$ could be predicted for all investigated temperatures and compositions of the solvent system decane/DMF in almost quantitative good agreement with the experimental data. The consistency of QC calculations, PC-SAFT predictions and experimental data speaks in favor of both, the correctness of theoretical approach as well as the quality of the experiments performed in this work.

Moreover, it was shown that the solvent effect on chemical-reaction equilibria $\left(K_{x}\right)$ can be qualitatively correct predicted only based on the standard Gibbs energies of reaction $\Delta^{R} g^{\infty}$ at infinite dilution obtained from both, QC calculations and PC-SAFT predictions, thus, significantly reducing the experimental effort required for finding the best solvent candidate. 


\section{AUTHOR INFORMATION}

\section{Corresponding Author}

*Email: Gabriele.Sadowski@bci.tu-dortmund.de

\section{Funding Sources}

Deutsche Forschungsgemeinschaft (DFG, German Research Foundation)

\section{Notes}

The authors declare no competing financial interest.

\section{ACKNOWLEDGMENT}

This work is part of the Collaborative Research Center/ Transregio 63 "Integrated Chemical Processes in Liquid Multiphase Systems" (subprojects A3 and A4). Financial support by the Deutsche Forschungsgemeinschaft (DFG, German Research Foundation) is gratefully acknowledged (TRR 63). We are also very grateful to Umicore AG and Co. KG for the supply of the catalyst precursor $\mathrm{Rh}(\mathrm{acac})(\mathrm{CO})_{2}$. 


\section{REFERENCES}

1. Reichardt C, Welton T. Solvents and Solvent Effects in Organic Chemistry. Weinheim: $\mathrm{VCH}, 2010$.

2. Schäfer E, Brunsch Y, Sadowski G, Behr A. Hydroformylation of 1-Dodecene in the Thermomorphic Solvent System Dimethylformamide/Decane. Phase Behavior-Reaction Performance-Catalyst Recycling. Industrial \& Engineering Chemistry Research. 2012; 51: 10296-10306.

3. Wiese K-D, Obst D. Hydroformylation. In: Beller M. Catalytic Carbonylation Reactions. Berlin Heidelberg: Springer-Verlag, 2006:1-33.

4. Palo DR, Erkey C. Kinetics of the Homogeneous Catalytic Hydroformylation of 1Octene in Supercritical Carbon Dioxide with $\mathrm{HRh}(\mathrm{CO})[\mathrm{P}(\mathrm{p}-\mathrm{CF} 3 \mathrm{C} 6 \mathrm{H} 4) 3] 3$. Industrial \& Engineering Chemistry Research. 1999; 38: 3786-3792.

5. Hemminger O, Marteel A, Mason MR, Davies JA, Tadd AR, Abraham MA. Hydroformylation of 1-hexene in supercritical carbon dioxide using a heterogeneous rhodium catalyst. 3. Evaluation of solvent effects. Green Chemistry. 2002; 4: 507-512.

6. Abu-Reziq R, Alper H, Wang D, Post ML. Metal Supported on Dendronized Magnetic Nanoparticles: Highly Selective Hydroformylation Catalysts. Journal of the American Chemical Society. 2006; 128: 5279-5282.

7. Haumann M, Riisager A. Hydroformylation in Room Temperature Ionic Liquids (RTILs): Catalyst and Process Developments. Chemical Reviews. 2008; 108: 14741497.

8. Deshpande RM, Divekar SS, Gholap RV, Chaudhari RV. Enhancement of rate and selectivity in hydroformylation of allyl alcohol through solvent effect. Industrial \& Engineering Chemistry Research. 1991; 30: 1389-1390.

9. Mieczyńska E, Trzeciak AM, Ziółkowski JJ. Effect of carboxylic acids on the yield and selectivity of the hydroformylation of hex-1-ene catalysed by [Rh(acac) (CO) (PPh3)]. Journal of Molecular Catalysis. 1993; 80: 189-200.

10. Monflier E, Bricout H, Hapiot F, Tilloy S, Aghmiz A, Masdeu-Bultó AM. HighPressure31P $\{1 \mathrm{H}\}$ NMR Studies of RhH(CO)(TPPTS)3 in the Presence of Methylated Cyclodextrins: New Light on Rhodium-Catalyzed Hydroformylation Reaction Assisted by Cyclodextrins. Advanced Synthesis \& Catalysis. 2004; 346: 425-431.

11. Kramarz KW, Klingler RJ, Fremgen DE, Rathke JW. Toroid NMR probes for the in situ examination of homogeneous cobalt hydroformylation catalysts at high pressures and temperatures. Catalysis Today. 1999; 49: 339-352.

12. De C, Saha R, Ghosh S, Ghosh A, Mukherjee K, Bhattacharyya S, Saha B. A review of biphasic hydroformylation for long chain substrates. Research on Chemical Intermediates. 2013; 39: 3463-3474.

13. Markert J, Brunsch Y, Munkelt T, Kiedorf G, Behr A, Hamel C, Seidel-Morgenstern A. Analysis of the reaction network for the Rh-catalyzed hydroformylation of 1-dodecene in a thermomorphic multicomponent solvent system. Applied Catalysis A: General. 2013; 462-463: 287-295.

14. Kiedorf G, Hoang DM, Müller A, Jörke A, Markert J, Arellano-Garcia H, SeidelMorgenstern A, Hamel C. Kinetics of 1-dodecene hydroformylation in a thermomorphic solvent system using a rhodium-biphephos catalyst. Chemical Engineering Science. 2014; 115: 31-48. 
15. Kegl T. Computational aspects of hydroformylation. RSC Advances. 2015; 5: 4304-4327.

16. Rush LE, Pringle PG, Harvey JN. Computational Kinetics of Cobalt-Catalyzed Alkene Hydroformylation. Angewandte Chemie. 2014; 126: 8816-8820.

17. Decker SA, Cundari TR. DFT Study of the Ethylene Hydroformylation Catalytic Cycle Employing a HRh(PH3)2(CO) Model Catalyst. Organometallics. 2001; 20: 2827-2841.

18. Cramer CJ, Truhlar DG. Implicit Solvation Models: Equilibria, Structure, Spectra, and Dynamics. Chemical Reviews. 1999; 99: 2161-2200.

19. Gross J, Sadowski G. Perturbed-Chain SAFT: An Equation of State Based on a Perturbation Theory for Chain Molecules. Ind. Eng. Chem. Res. 2001; 40: 1244-1260.

20. Uyan M, Sieder G, Ingram T, Held C. Predicting CO2 solubility in aqueous Nmethyldiethanolamine solutions with ePC-SAFT. Fluid Phase Equilibria. 2015; 393: 91100.

21. Riechert O, Husham M, Sadowski G, Zeiner T. Solvent effects on esterification equilibria. AIChE J. 2015; 61: 3000-3011.

22. Barker JA, Henderson D. Perturbation Theory and Equation of State for Fluids: The Square-Well Potential. The Journal of Chemical Physics. 1967; 47: 2856-2861.

23. Gross J, Sadowski G. Application of the Perturbed-Chain SAFT Equation of State to Associating Systems. Ind. Eng. Chem. Res. 2002; 41: 5510-5515.

24. McQuarrie DA, Simon JD. Physical Chemistry: A Molecular Approach: University Science Books, 1997.

25. Klamt A, Schuurmann G. COSMO: a new approach to dielectric screening in solvents with explicit expressions for the screening energy and its gradient. Journal of the Chemical Society, Perkin Transactions 2. 1993: 799-805.

26. Zhou H-X, Gilson MK. Theory of Free Energy and Entropy in Noncovalent Binding. Chemical Reviews. 2009; 109: 4092-4107.

27. Becke AD. Density-functional exchange-energy approximation with correct asymptotic behavior. Physical Review A. 1988; 38: 3098-3100.

28. Perdew JP. Density-functional approximation for the correlation energy of the inhomogeneous electron gas. Physical review B. 1986; 33: 8822.

29. Lee C, Yang W, Parr RG. Development of the Colle-Salvetti correlation-energy formula into a functional of the electron density. Physical review B. 1988; 37: 785.

30. Møller C, Plesset MS. Note on an approximation treatment for many-electron systems. Physical Review. 1934; 46: 618.

31. Riplinger C, Neese F. An efficient and near linear scaling pair natural orbital based local coupled cluster method. The Journal of Chemical Physics. 2013; 138: 034106.

32. Schäfer A, Huber C, Ahlrichs R. Fully optimized contracted Gaussian basis sets of triple zeta valence quality for atoms Li to Kr. The Journal of Chemical Physics. 1994; 100: 5829-5835.

33. Dunning Jr TH. Gaussian basis sets for use in correlated molecular calculations. I. The atoms boron through neon and hydrogen. The Journal of Chemical Physics. 1989; 90: 1007-1023.

34. Kendall RA, Dunning Jr TH, Harrison RJ. Electron affinities of the first-row atoms revisited. Systematic basis sets and wave functions. The Journal of Chemical Physics. 1992; 96: 6796-6806. 
35. Grimme S, Antony J, Ehrlich S, Krieg H. A consistent and accurate ab initio parametrization of density functional dispersion correction (DFT-D) for the 94 elements H-Pu. The Journal of Chemical Physics. 2010; 132: 154104.

36. Weigend F. Accurate Coulomb-fitting basis sets for $\mathrm{H}$ to Rn. Physical chemistry chemical physics. 2006; 8: 1057-1065.

37. TURBOMOLE V6.4 2012, a development of University of Karlsruhe and Forschungszentrum Karlsruhe GmbH, 1989-2007, TURBOMOLE GmbH, since 2007; available from $\mathrm{http}: / / \mathrm{www}$. turbomole.com

38. Neese F. The ORCA program system. Wiley Interdisciplinary Reviews: Computational Molecular Science. 2012; 2: 73-78.

39. Vogelpohl C, Brandenbusch C, Sadowski G. High-pressure gas solubility in multicomponent solvent systems for hydroformylation. Part II: Syngas solubility. The Journal of Supercritical Fluids. 2014; 88: 74-84.

40. Vogelpohl C, Brandenbusch C, Sadowski G. High-pressure gas solubility in multicomponent solvent systems for hydroformylation. Part I: Carbon monoxide solubility. The Journal of Supercritical Fluids. 2013; 81: 23-32.

41. Yaws CL. Chemical Properties Handbook (1st edition). New York: McGraw-Hill, 1999.

42. Liessmann G, Schmidt W, Reiffarth S. Recommended Thermophysical Data. Data compilation of the Saechsische Olefinwerke. Boehlen, 1995.

43. Steele WV, Chirico RD. Thermodynamic Properties of Alkenes (Mono-Olefins Larger than C4). Journal of Physical and Chemical Reference Data. 1993; 22: 377-430.

44. Barin I, Platzki G. Thermochemical data of pure substances (3rd edition). Weinheim, New York: Wiley-VCH Verlag GmbH, 1995. 


\section{TABLES}

Table 1. Experimentally-determined pressures at reaction equilibrium $\left(p_{\text {eq }}\right)$, equilibrium compositions, $K_{x}$ and corresponding standard deviations for the HYFO of 1-dodecene to $n$-tridecanal at different temperatures and at varying solvent compositions.

\begin{tabular}{|c|c|c|c|c|c|c|c|c|c|c|c|c|}
\hline$T$ & $w_{\text {decane }} / w_{D M F}$ & $p_{e q}$ & $x_{C O}^{(l)}$ & $x_{H_{2}}^{(l)}$ & $x_{1-\text { dodecene }}^{(l)}$ & $x_{n-t r i d e c a n a l}^{(l)}$ & $x_{\text {iso-dodecenes }}^{(l)}$ & $x_{\text {iso-aldehydes }}^{(l)}$ & $x_{\text {dodecane }}^{(l)}$ & $x_{\text {decane }}^{(l)}$ & $x_{D M F}^{(l)}$ & $K_{x}$ \\
\hline$[\mathrm{K}]$ & [wt-\%/wt-\%] & [bar] & $\cdot 10^{3}[-]^{\mathrm{a}}$ & $10^{3}[-]^{\mathrm{a}}$ & $\cdot 10^{3}[-]^{\mathrm{b}}$ & $10^{3}[-]^{b}$ & $\cdot 10^{3}[-]^{\mathrm{b}}$ & $\cdot 10^{3}[-]^{\mathrm{b}}$ & $\cdot 10^{3}[-]^{\mathrm{b}}$ & $\cdot 10^{3}[-]^{\mathrm{b}}$ & $10^{3}[-]^{\mathrm{b}}$ & $\cdot 10^{-8}[-]$ \\
\hline \multirow[t]{3}{*}{368} & $80 / 20$ & $0.40 \pm 0.02$ & 0.188 & 0.102 & 1.78 & 61.03 & 82.27 & 0.97 & 2.84 & 572.28 & 278.53 & $18.69 \pm 4.36$ \\
\hline & $60 / 40$ & $0.35 \pm 0.02$ & 0.113 & 0.060 & 1.07 & 53.34 & 68.19 & 0.83 & 2.15 & 380.45 & 493.79 & $76.34 \pm 18.67$ \\
\hline & $0 / 100$ & $0.29 \pm 0.02$ & 0.044 & 0.023 & 0.83 & 33.82 & 43.18 & 0.47 & 1.10 & 0.00 & 920.52 & $441.52 \pm 137.24$ \\
\hline \multirow[t]{3}{*}{378} & $80 / 20$ & $0.74 \pm 0.07$ & 0.415 & 0.230 & 0.79 & 55.09 & 86.65 & 2.36 & 3.78 & 572.21 & 278.48 & $7.64 \pm 1.58$ \\
\hline & $60 / 40$ & $0.58 \pm 0.04$ & 0.221 & 0.121 & 0.89 & 48.68 & 72.30 & 1.13 & 2.65 & 380.39 & 493.64 & $20.92 \pm 1.71$ \\
\hline & $0 / 100$ & $0.57 \pm 0.03$ & 0.115 & 0.061 & 0.43 & 29.08 & 47.19 & 1.30 & 1.46 & 0.00 & 920.37 & $99.02 \pm 12.04$ \\
\hline \multirow[t]{3}{*}{388} & $80 / 20$ & $1.11 \pm 0.07$ & 0.654 & 0.372 & 1.15 & 58.89 & 82.81 & 1.90 & 4.10 & 571.85 & 278.28 & $2.18 \pm 0.39$ \\
\hline & $60 / 40$ & $0.98 \pm 0.05$ & 0.425 & 0.238 & 0.67 & 47.10 & 72.65 & 1.82 & 3.42 & 380.23 & 493.44 & $7.02 \pm 0.58$ \\
\hline & $0 / 100$ & $0.81 \pm 0.08$ & 0.166 & 0.090 & 0.46 & 29.53 & 46.32 & 1.15 & 1.88 & 0.00 & 920.40 & $45.43 \pm 11.15$ \\
\hline
\end{tabular}

${ }^{\mathrm{a}}$ Calculated using PC-SAFT (Eq. 1 ), ${ }^{\mathrm{b}}$ Relative standard deviation $<1 \%$ 
Table 2. Reaction enthalpy $\Delta^{R} h^{0}$ and Gibbs energy of reaction $\Delta^{R} g^{0}$ for the HYFO of 1dodecene to $n$-tridecanal at the ideal-gas standard state $(\mathrm{p}=1.013 \mathrm{bar})$ as obtained in this work applying different methods.

\begin{tabular}{ccccc}
\hline $\begin{array}{c}\text { Thermochemical } \\
\text { quantity }\end{array}$ & $T[\mathrm{~K}]$ & obtained by & $\begin{array}{c}\text { value } \\
{[\mathrm{kJ} / \mathrm{mol}]}\end{array}$ & $\begin{array}{c}\text { estimated } \\
\text { uncertainty } \\
{[\mathrm{kJ} / \mathrm{mol}]}\end{array}$ \\
\hline & 373 & Eq. $3^{\mathrm{a}}$ & -117.00 & \pm 5.49 \\
& & MP2/def2-TZVP & -123.14 & \pm 4.00 \\
\cline { 2 - 5 }$\Delta^{R} h^{0}$ & 383 & Eq. 3 & -117.15 & \pm 5.49 \\
& & MP2/def2-TZVP & -123.33 & \pm 4.00 \\
\hline \multirow{2}{*}{378} & Eq. 3 & -20.01 & \pm 5.65 \\
$\Delta^{R} g^{0}$ & & MP2/def2-TZVP & -17.86 & \pm 4.00 \\
\end{tabular}

${ }^{\mathrm{a}}$ Values for properties of formation taken from literature ${ }^{41-44}$

Table 3. Standard Gibbs energy of reaction $\Delta^{R} g^{\infty}$ at infinite dilution in liquid solvents for the HYFO of 1-dodecene to $n$-tridecanal at $378.15 \mathrm{~K}$ obtained from MP2/COSMO calculations and from MP2/PC-SAFT

\begin{tabular}{cccc}
\hline $\begin{array}{c}w_{\text {decane }} / w_{D M F} \\
{[\mathrm{wt}-\% / \mathrm{wt}-\%]}\end{array}$ & $\mathrm{p}$ & $\Delta^{R} g^{\infty}(\mathrm{MP} 2 / \mathrm{COSMO})$ & $\Delta^{R} g^{\infty}(\mathrm{MP} 2 / \mathrm{PC}-\mathrm{SAFT})$ \\
\hline $80 / 20$ & 0.74 & {$[\mathrm{~kJ} / \mathrm{mol}]$} & {$[\mathrm{kJ} / \mathrm{mol}]$} \\
$0 / 100$ & 0.57 & -36.48 & -68.76 \\
\hline
\end{tabular}


Table 4. Thermodynamic equilibrium constants for the HYFO of 1 -dodecene to $n$-tridecanal at the ideal-gas standard state $(p=1.013$ bar) and at different temperatures obtained from MP2 calculations $\left(K_{f}\right)$ and from experimental data combined with PC-SAFT $\left(K_{f, \text { exp }}\right)$

\begin{tabular}{ccc}
\hline$T[\mathrm{~K}]$ & $K_{f}[-]$ & $K_{f, \exp }[-]$ \\
\hline 368 & 848.85 & 973.08 \\
378 & 292.97 & 322.82 \\
388 & 106.65 & 129.34 \\
\hline
\end{tabular}


Table 5. PC-SAFT predicted mole fractions and fugacity coefficients of reactants and products at equilibrium for the HYFO of 1dodecene to $n$-tridecanal (to be compared with the experimental results given in Table 1).

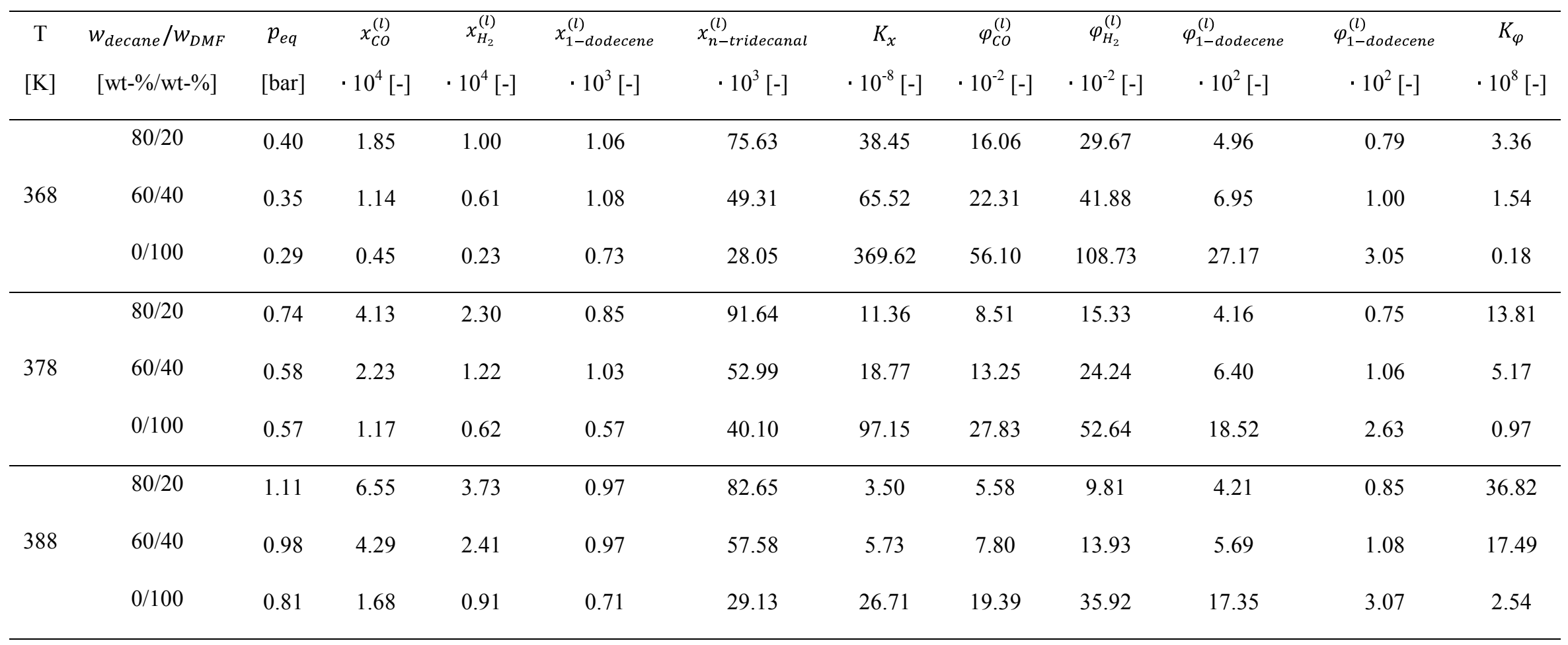




\section{FIGURES}

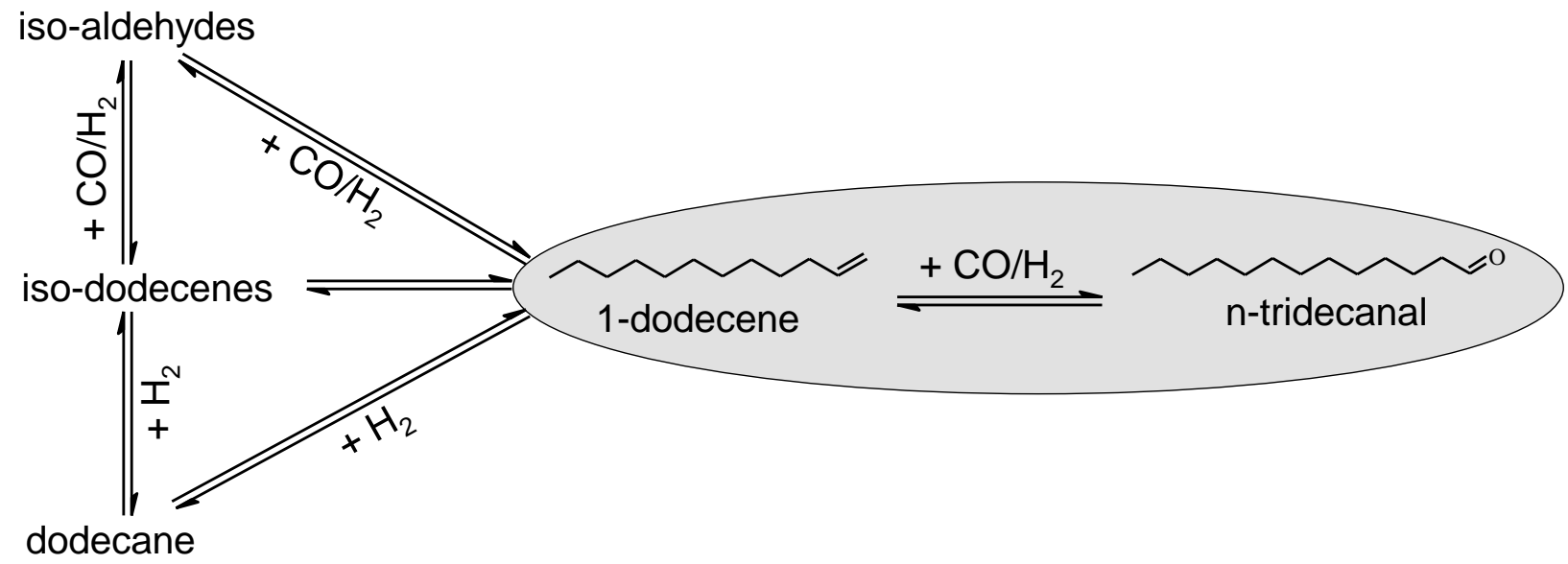

Figure 1. Reaction network for the Rh-catalyzed HYFO of 1-dodecene.

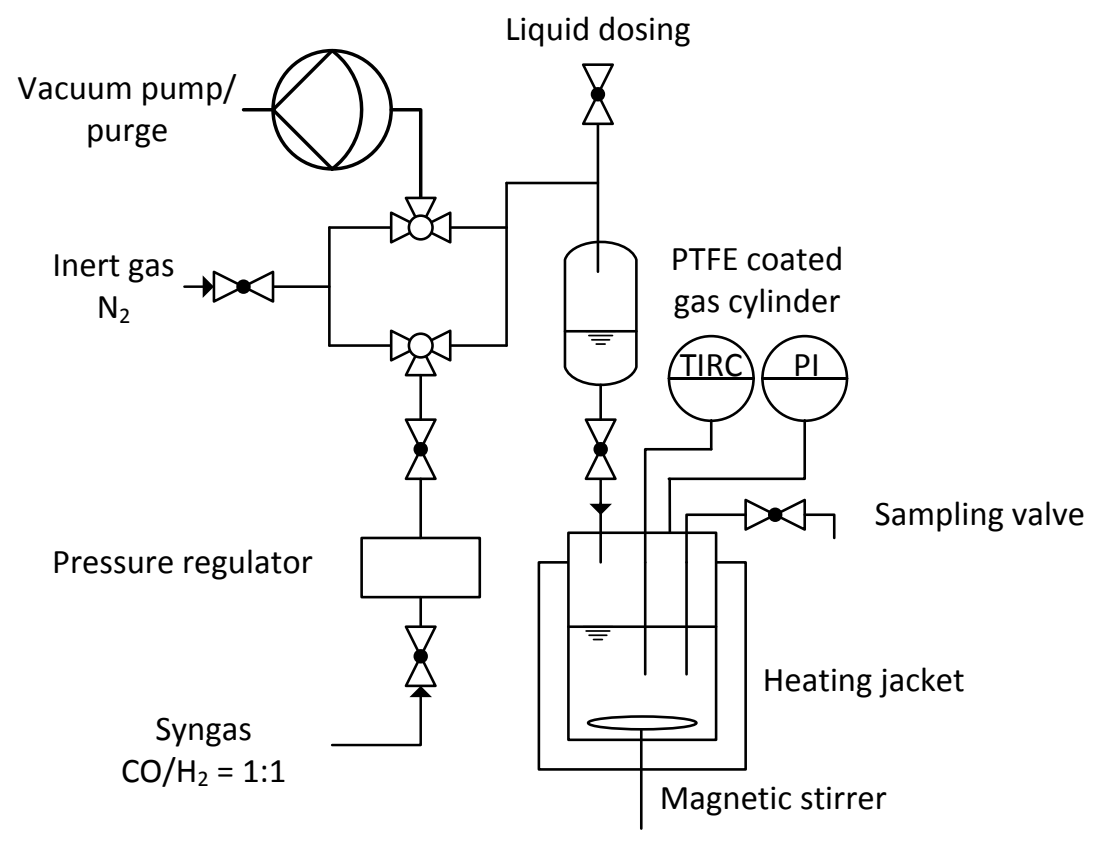

Figure 2. Scheme of experimental apparatus for separate catalyst pretreatment and substrate injection using a PTFE-coated gas cylinder. 


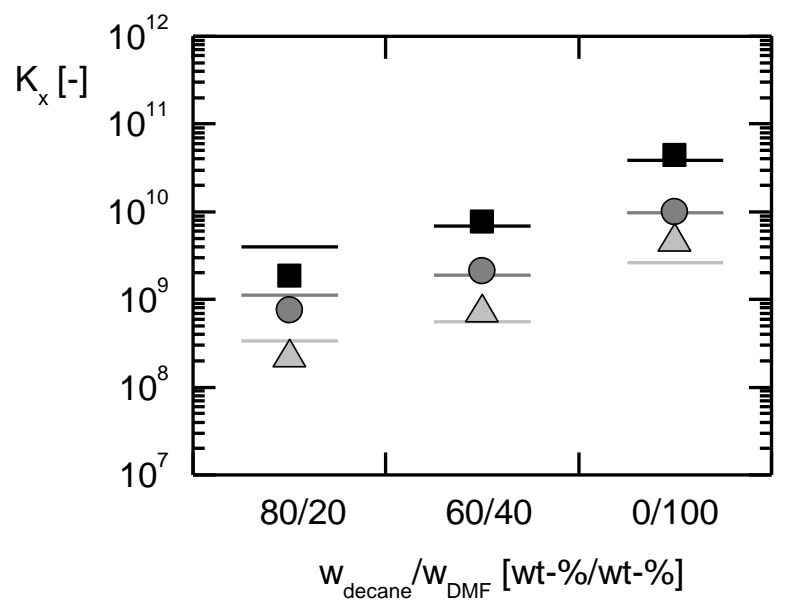

Figure 3. $K_{x}$ values for the HYFO of 1-dodecene to $n$-tridecanal at different compositions of the decane/DMF solvent system. The symbols are experimental data at $368 \mathrm{~K}$ (black squares), $378 \mathrm{~K}$ (dark grey circles) and $388 \mathrm{~K}$ (light grey triangles) as reported in Table 1. All error bars are smaller than the symbols and are therefore not shown. The lines mark purely-predicted values from PC-SAFT presented in Table 5. 